\title{
A survey of transcriptome complexity using PacBio single-molecule real-time analysis combined with Illumina RNA sequencing for a better understanding of ricinoleic acid biosynthesis in Ricinus communis
}

\author{
Lijun Wang ${ }^{1}$, Xiaoling Jiang ${ }^{3}$, Lei Wang ${ }^{1}$, Wei Wang ${ }^{1}$, Chunling Fu' ${ }^{1}$ Xingchu Yan ${ }^{1 *}$ and Xinxin Geng ${ }^{2^{*}}$
}

\begin{abstract}
Background: Ricinus communis is a highly economically valuable oil crop plant from the spurge family, Euphorbiaceae. However, the available reference genomes are incomplete and to date studies on ricinoleic acid biosynthesis at the transcriptional level are limited.

Results: In this study, we combined PacBio single-molecule long read isoform and Illumina RNA sequencing to identify the alternative splicing (AS) events, novel isoforms, fusion genes, long non-coding RNAs (IncRNAs) and alternative polyadenylation (APA) sites to unveil the transcriptomic complexity of castor beans and identify critical genes related to ricinoleic acid biosynthesis. Here, we identified 11,285 AS-variants distributed in 21,448 novel genes and detected 520 fusion genes, 320 IncRNAs and 9511 (APA-sites). Furthermore, a total of 6067, 5983 and 4058 differentially expressed genes between developing beans of the $R$. communis lines 349 and 1115 with extremely different oil content were identified at 7, 14 and 21 days after flowering, respectively. Specifically, 14, 18 and 11 DEGs were annotated encoding key enzymes related to ricinoleic acid biosynthesis reflecting the higher castor oil content of 1115 compared than 349. Quantitative real-time RT-PCR further validated fifteen of these DEGs at three-time points.

Conclusion: Our results significantly improved the existed gene models of $R$. communis, and a putative model of key genes was built to show the differences between strains 349 and 1115, illustrating the molecular mechanism of castor oil biosynthesis. A multi-transcriptome database and candidate genes were provided to further improve the level of ricinoleic acid in transgenic crops.
\end{abstract}

Keywords: Ricinus communis, Full-length transcriptome, Illumina RNA sequencing, Ricinoleic acid biosynthesis, Key enzymes

\section{Background}

Ricinus communis ( $R$. communis) L., is a highly economically valuable oil plant from the spurge family, Euphorbiaceae, and arguably one of the non-edible oil crops with the highest application value. Recently, with the

\footnotetext{
* Correspondence: yanxc@oilcrops.cn; creamxinxin111@sina.com

'Oil Crops Research Institute of the Chinese Academy of Agricultural

Sciences/Key Laboratory of Biology and Genetic, Improvement of Oil Crops, Ministry of Agriculture, Wuhan, China

${ }^{2}$ Applied Biotechnology Center, Wuhan University of Bioengineering, Wuhan, China

Full list of author information is available at the end of the article
}

looming shortage of non-renewable resources such as oil and natural gas, castor oil has attracted worldwide attention as an alternative renewable resource [1]. However, the previously reported reference genomes are incomplete and had limited annotation and structural information. Although the sequence information of several species obtained through short-read sequencing has been published in recent years [2-4], the knowledge on fulllength (FL) sequences of mRNAs remains limited, especially in $R$. communis. FL transcripts can significantly improve the accuracy of genome annotation/ assembly

(c) The Author(s). 2019 Open Access This article is distributed under the terms of the Creative Commons Attribution 4.0 International License (http://creativecommons.org/licenses/by/4.0/), which permits unrestricted use, distribution, and reproduction in any medium, provided you give appropriate credit to the original author(s) and the source, provide a link to the Creative Commons license, and indicate if changes were made. The Creative Commons Public Domain Dedication waiver (http://creativecommons.org/publicdomain/zero/1.0/) applies to the data made available in this article, unless otherwise stated. 
and transcriptome information, which helps to identify full length isoforms of a gene, alternative splicing (AS) events, long non-coding RNAs (lncRNAs) and alternative polyadenylation (APA) sites [5].

Next-generation sequencing (NGS; i.e., Illumina RNA sequencing) can generate digital data on gene expression without the limits of predesigned probes [6], and has been conducted not only to create the reference genomes such as in Arabidopsis [7], rice [8], maize [9], but also provides the de-novo assembly for many organisms in the past including animals and plants $[10,11]$. The results of incomplete (ranging between 1 and $2 \mathrm{~kb}$, including a methylated cap at the $5^{\prime}$ end and poly- $\mathrm{A}$ tails at the $3^{\prime}$ end) and low-quality transcripts obtained through Illumina RNA sequencing limit the scope of analysis of alternative splicing variants and corrected annotation [12]. However, the advent of Pacific Biosciences (PacBio) single-molecule long read isoform sequencing (SMRTseq) technology has enabled us to obtain long-read or full-length transcriptomes, which allows the collection of large-scale long-read transcripts with complete coding sequences and characterization of gene families (the average read length of PacBio SMRT-seq is $>10 \mathrm{~kb}$ and the real length can be up to $60 \mathrm{~kb}$ ) [13-16]. However, it was reported that SMRT-seq provided inaccurate information on genes, less coverage of genes led to the high error rate, which could be corrected using Illumina RNA sequencing reads and circular-consensus (CCS) reads [17]. Recently, the strategy of combining SMRT-seq and Illumina RNA-seq has been applying to generate comprehensive information, detect more gene isoforms and reveal functional variety etc., at the transcriptional level, which consummated the genome database offers a scientific basis for molecular breeding $[16,18]$.

Castor oil is called a "renewable green oil", with an annual global production as high as 500,000 tons. Developing castor oil resources is one of the most important technical ways to reduce oil dependence, maintain the ecological balance and can enhance rapid economic growth [19]. The main component of castor oil is triglycerides of higher fatty acids. Eight different fatty acids found in castor oil, among, ricinoleic (66\%), oleic (8\%), palmitic $(8 \%)$ and linoleic acid (6\%) were the major components [19]. The content of unsaturated fatty acids was as high as $88 \%$, which gives castor oil good prospects for development and application [19].

The biosynthetic pathway of castor oil had earlier discovered by Lin et al. [19]. Oleoyl-ACP is first synthesized in plastids, then hydrolyzed to free oleic acids by oleoyl-ACP thioesterase (AAT), and finally converted by acyl-CoA synthase (ACS) to form oleoyl-CoA [20]. Subsequently, oleoyl$\mathrm{CoA}$ is exported into the acyl pool and used as the raw material for the synthesis of ricinolate. Oleoyl-CoA enters the endoplasmic reticulum and the acyl editing pathway, in which lysophosphatidylcholine acyltransferase (LPCAT) transfers the oleoyl group of oleoyl-CoA to the sn-2 site of phosphatidylcholine (PC) as the substrate of oleoyl-12hydroxylase (FAH12), which catalyzes the conversion of 2oleoyl-PC to 2-ricinoleoyl-PC. Phospholipase A2 (PLA2) releases ricinoleate and lysophosphatidylcholine (LPC) from 2-ricinoleoyl-PC simultaneously. LPC can form sn-2oleoyl-PC again as the substrate of FAH12, in a reaction catalyzed by LPCAT. Ricinoleate is converted into ricinoleoyl-CoA by long-chain lipoyl-CoA synthetase (LACS), which acts as an acyl donor that enters the acylCoA-dependent pathway, and finally TAG is formed by acylation reactions. The glycerol-3-phosphate acyltransferase (GPAT) first forms ricinoleyl-lysoPA. Lysophosphatidic acid acyltransferase (LPAAT), the second acyltransferase enzyme catalyzes the acylation of the LPA site to produce phosphatidic acid (PA). PA is phosphorylated by phosphatidic acid phosphatase (PAP) to yield diacylglycerol (DAG). Diacylglycerol acyltransferase (DGAT), the third acyltransferase, catalyzes the acylation of the sn-3 site of DAG to form TAG. The fatty acid specificity of these acyltransferases determines the composition of fatty acids in the TAGs. The key enzymatic steps are catalyzed by acyl-ACP thioesterase, lysophosphatidylcholine acyltransferase, oleoyl-12-hydroxylase, phospholipase A2, diacylglycerol acyltransferase and a ratio of phosphatidylcholine:diacylglycerol acyltransferase. Furthermore, Lin at al. (2007) reported that phospholipase C2 (PLC2) catalyzes the conversion of 2-oleoyl-PC into 1-acyl-2-oleoyl-sn-glycerol. It may prevent the incorporation of hydroxy fatty acids into TAG and increase the content of ricinoleic acid in the seeds [19]. Apart from acyl-CoA-dependent pathway, acyl-CoAindependent pathway can also produce TAGs.

This study firstly unveils the full-length transcriptome sequences from $R$. communis obtained through the PacBio SMRT technique compare with Illumina RNA sequencing to generate a more complete transcriptome, and further to detect AS-variants, fusion genes, IncRNAs and APAsites. Illumina RNA-seq was also used in this study to improve the PacBio SMRT transcript isoforms through short-read error correction and comparison of the differences between transcripts from the two platforms. In addition, Illumina RNA-seq transcriptome analysis of the immature seeds of two $R$. communis lines with extremely different castor oil contents, 349 and 1115 , was used to identify the genes related to ricinoleic acid biosynthesis. Our results have the potential to improve $R$. communis genome existing model, contribute towards understanding of the complexity of the $R$. communis genome, and serve as reference sequences for differential expression analysis in the future. The annotation of DEGs between two varieties with extremely different levels of oil content will help further understanding of the detailed mechanism of ricinoleic acid biosynthesis in $R$. communis. 


\section{Results}

\section{PacBio SMRT sequencing}

To better identify full-length splice variants, novel genes, APA-sites etc., PacBio Iso-Seq platform was applied to sequence the transcriptome of $R$. communis on the basis of the Illumina HiSeqTM 2500 sequencing platform (Illumina Inc., San Diego, CA, US). A total of 3 cells (1-2 kb, $2-3 \mathrm{~kb}$ and $3-6 \mathrm{~kb}$ ) were constructed to eliminate bias of the instrument for short fragments (Fig. 1a, b). A dataset with $9.42 \mathrm{~Gb}$ of clean reads was obtained after filtering using SMRTLink (4.0). The mean length of clean reads in the three libraries was between 1698 and 4132 bp (Additional file 1: Table S1). A total of 242,942 reads of insert (ROI) were screened with full passes $\geq 0$ and accuracy of sequence was set at $\geq 0.75$. The mean length of the ROI in the three libraries was between 2088 and 4013 bp (Additional file 1: Table S2, Additional file 2: Figure S1). The number of full-length reads, which contained poly-A, 5' and 3' primers, was 111,507 (45.90\% of total ROI). There were 110,469 full-length non-chimeric reads with a mean length of $2201 \mathrm{bp}$ (Additional file 1: Table S3). In total, 50,636 high-quality isoforms were identified (Additional file 1: Table S4). The isoform lengths of the PacBio SMRT sequences were longer than those of the reference genome (Fig. 1c), which indicated that more full-length and novel isoforms were identified based on PacBio SMRT sequencing. Mapping the results based on the reference genome revealed that a total of $20.97 \%$ of the isoforms detected by PacBio SMRT sequencing were the same with the corresponding genomic annotations, $1.74 \%$ of all transcripts were partially mapped to the genome, and $62.00 \%$ potentially were novel isoforms (Fig. 1d). The 20 longest scaffolds (Fig. 2a) of $R$. communis genome were selected to compare the isoforms density. Thus, the result showed that PacBio SMRT sequencing data had higher gene (Fig. 2b, d) and transcript density (Fig. 2c, e) than the $R$. communis reference genome. Consequently, the $R$. communis genome was enriched with the PacBio SMRT results and used for further analysis.

\section{Detection of alternative splicing events and novel genes}

Pre-mRNA, a precursor in eukaryotic gene transcription, has multiple splicing patterns. Five main AS events (alternative $3^{\prime}$ splice sites, alternative $5^{\prime}$ splice sites, retained introns, skipped exons and mutually exclusive exons) were identified. A total of 11,285 AS events were found in $R$. communis based on the PacBio SMRT reads. The number of three kinds of events, alternative 3' splice sites (2177), alternative $5^{\prime}$ splice sites (1063) and retained introns (6890), were much higher than those of
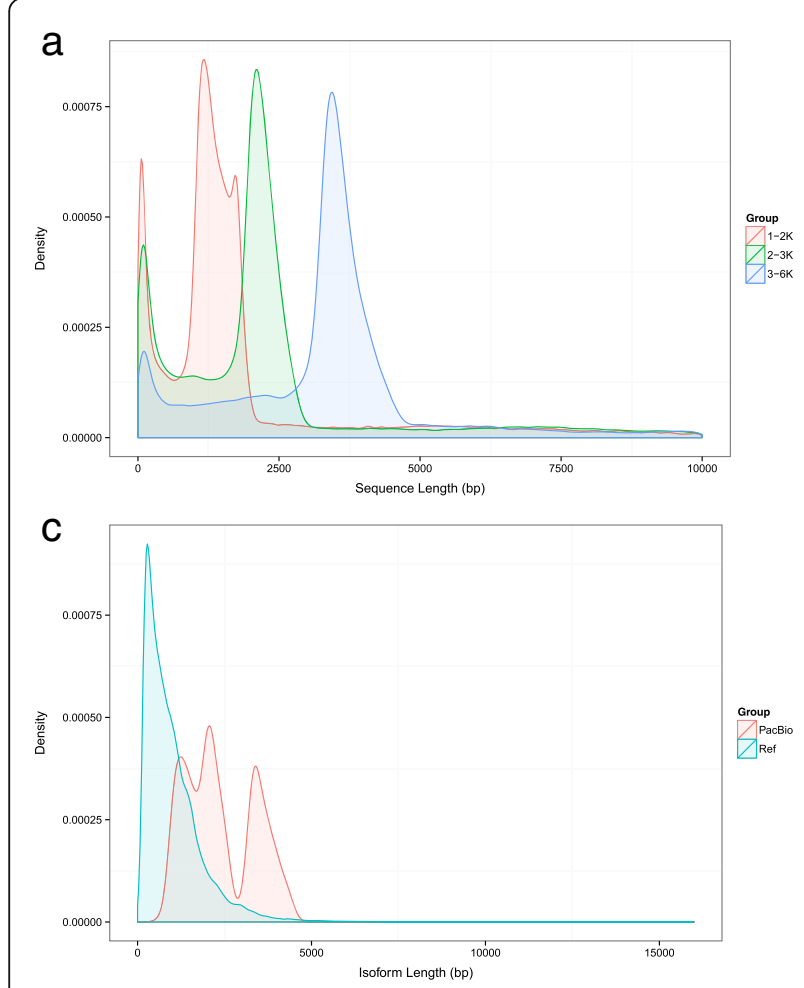

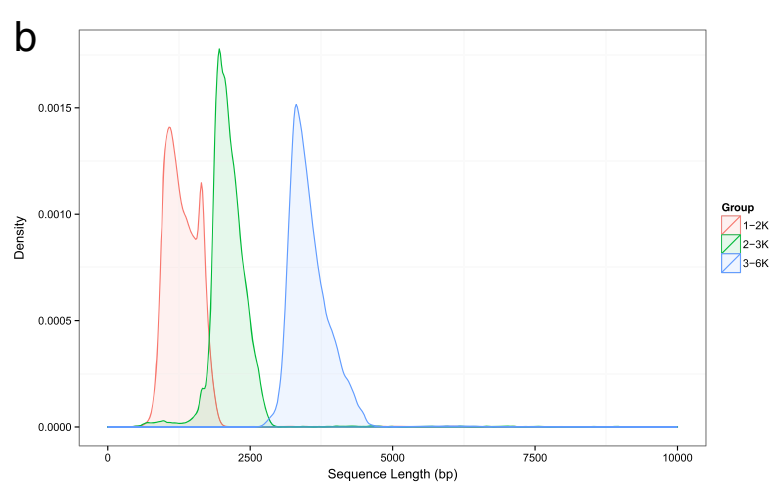

d

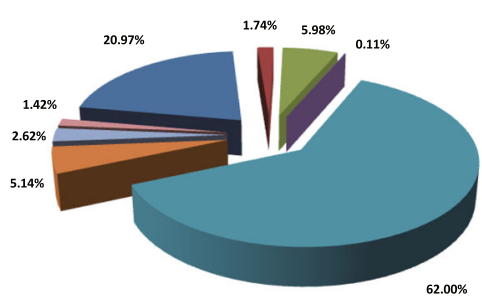

Fig. 1 Library construction of PacBio SMRT sequencing and isoform comparison between the Ricinus communis genome and full-length transcriptome. a Quality inspection of reads of inserts (ROI) in three libraries (1-2k, 2-3k and 3-6 k). b Quality inspection of full-length non-chimeric (FLNC) reads in three libraries (1-2 k, 2-3 k and 3-6 k). $\mathbf{c}$ Isoform length comparison between the reference genome and PacBio long-reads data. $\mathbf{d}$ Comparison of isoforms sequences between the Ricinus communis genome and full-length transcriptome 


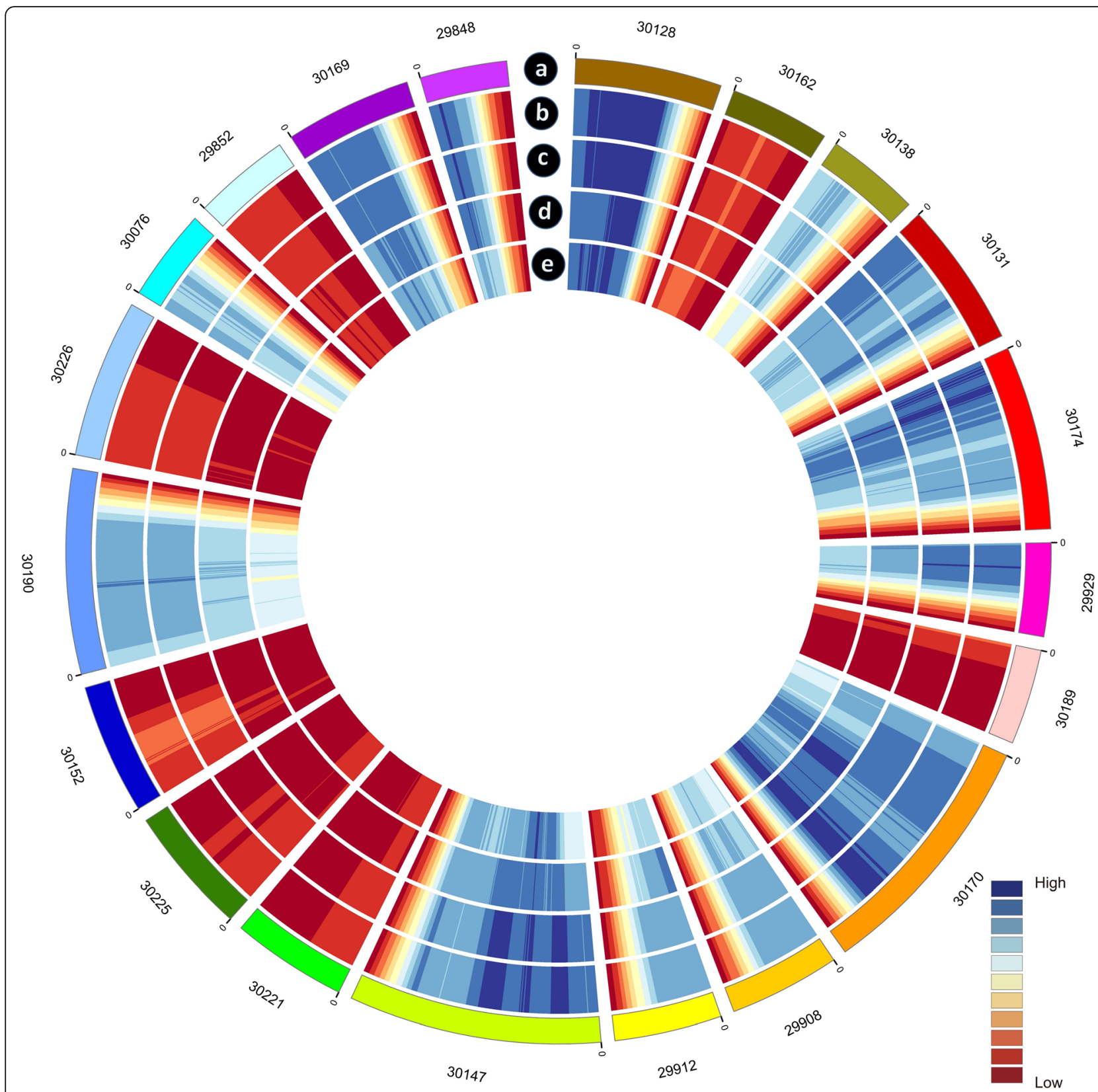

Fig. 2 CIRCOS visualization of gene and transcript density compared PacBio SMRT sequences with Ricinus communis reference genome for 20 top lengths of scaffolds. a Twenty longest scaffolds schematic. $\mathbf{b}$ Heat map of gene density distribution of PacBio SMRT sequences. Gene density was calculated in a $1-M b$ sliding window at $20 \mathrm{~kb}$ intervals. $\mathbf{c}$ Heat map of transcripts density distribution of PacBio SMRT sequences. Gene density was calculated in a 1-Mb sliding window at $20 \mathrm{~kb}$ intervals. $\mathbf{d}$ Heat map of gene density distribution of the reference genome. Gene density was calculated in a 1-Mb sliding window at $20 \mathrm{~kb}$ intervals. e Heat map of transcripts density distribution of the reference genome. Gene density was calculated in a 1-Mb sliding window at $20 \mathrm{~kb}$ intervals

skipped exons (1056) and mutually exclusive exons (99). Among these, a new gene PB.2740 had the largest number of splice variants ( 23 transcripts) according to the PacBio SMRT sequencing data. There were no significant differences among other plants in the distribution of AS events, with the majority of AS events being retained introns (Fig. 3a). To some extent, alternative splicing allows the same gene to produce multiple isoforms. Isoform numbers from PacBio SMRT reads are summarized in Fig. 3b. As the reference genome annotations are not accurate, it is necessary to optimize the structure of the original annotated genes. If mapped reads support in areas outside the original gene boundaries, we extended the gene's untranslated region (UTR) 


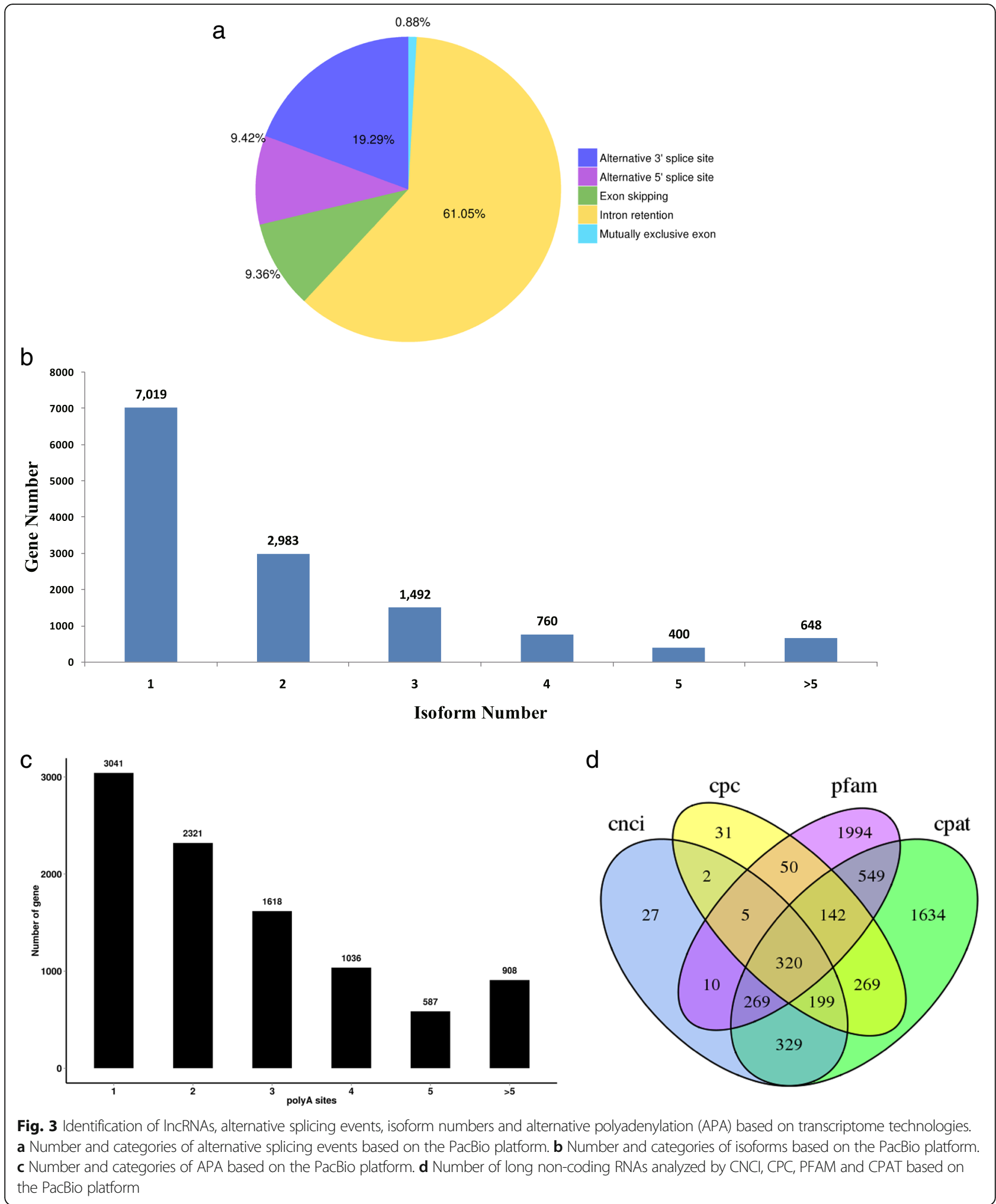

upstream and downstream to correct the gene boundary. The gene structure optimization results are shown in Additional file 1: Table S5. The Prediction of novel genes may enrich the genome information of $R$. communis and offer a new direction to further study ricinoleic acid biosynthesis. A total of 21,448 novel genes were identified using PacBio SMRT sequencing approach and 98.48\% $(21,122)$ were annotated according to information from 
the Cluster of Orthologous Groups (COG) of proteins [21] (8991), the gene ontology (GO) [22] $(15,841)$, the Kyoto Encyclopedia of Genes and Genomes (KEGG) [23] (9267), the protein family (Pfam) [24] $(16,822)$, the manually annotated and reviewed protein sequence database (SwissProt) [25] $(15,188)$ and Non-redundant protein (NR) [26] $(21,112)$ databases (Additional file 1: Table S6).

\section{Identification of fusion genes, alternative polyadenylation, transcription factor and LncRNAs}

A fusion gene is a chimeric gene composed of two or more separate genes [5]. A total of 250 fusion genes were identified in the SMRT-seq library. The detailed information of 250 fusion genes was supplied in Additional file 1: Table S7. Our results revealed that all fusion events were comprised of two genes, which was consistent with previous reports that fusion events mostly involve two genes [27]. In eukaryotes, APA of pre-mRNA may contribute to transcriptome diversity, genome coding capacity and gene regulation. A total of 9511 APA genes were identified using the TAPIS pipeline. Among these, 3041 genes (36.15\%) with a single poly-A site were detected, and the number of genes with two or more poly-A sites was 5562. For 908 genes, five or more poly-A sites were detected (Fig. 3c). Transcription factors (TFs) account for a large part of the plant genome and play a vital part in gene regulation. In our study, a total of 1356 genes were annotated as encoding TFs from 69 families according to SMRT-seq. Using our data, we identified novel genes from 58 families, increasing the number of TF isoforms to 2335 (Additional file 1: Table S8). Because lncRNAs do not encode proteins, the transcript can be screened to determine whether it has potential of coding ability. If one has no potential coding ability, it would be categorized as a bona-fide lncRNA. Four coding potential analysis methods were used to predict the lncRNAs among the novel transcripts. These methods included Coding Potential Calculator (CPC) [28], Coding-Non-Coding Index (CNCI) [29], Pfam [24] and Coding Potential Assessment Tool (CPAT) [30]. A total of 320 lncRNAs were found among all the datasets, including CPC (1018), CNCI (1161), CPAT (3711) and Pfam (3339) (Fig. 3d). Of the 320 lncRNAs, 154 lincRNAs, 57 antisense-lncRNAs, 15 intronic-lncRNAs and 56 sense-lncRNAs were identified. Moreover, the 20 longest scaffolds of $R$. communis genome were selected to study the fusion genes and lncRNAs identified by SMRT-seq. Mapping lncRNAs to the 20 longest scaffolds (Fig. 4a) revealed that they have similar distribution to that of protein-coding genes, which are enriched outside of pericentromeric regions (Fig. 4b). Ten fusion events of the 20 longest scaffolds by SMRT-seq were more likely to occur at inter-chromosomally (7) than intrachromosomally (3) (Fig. 4c).

\section{Illumina RNA sequencing}

To analyze the ricinoleic acid biosynthesis mechanism, we selected the two cultivars 349 and 1115 with extremely different castor oil content, and analyzed their different tissues at three-time points with triplicate condition for Illumina RNA-seq, resulting in a total of 18 libraries. Correlation analysis results of three replications of 18 samples revealed Pearson's correlation coefficients between 0.922 and 0.999 . Notably, all these values were greater than the 0.92 recommended under ideal experimental conditions (Additional file 2: Figure S2). A total of $121.50 \mathrm{~Gb}$ of clean data $(\mathrm{Q} 30>90.14 \%)$ was generated after filtering the original data, the GC contents were between 42.01 and 46.01\% (Additional file 1: Table S9). The error rate of all clean data per sample was controlled below $0.05 \%$. The amount of paired-end reads among the clean reads ranged from $20,165,714$ to 25 , 302,539 among the 18 samples, of which between 81.72 and $88.56 \%$ were successfully mapped to the reference genome. Of these mapped data, the percentage of samples that uniquely mapped to the genome was between 70.31 and $87.53 \%$ (Additional file 1: Table S10).

\section{Analysis of the DEGs}

DESeq software [31] was used to analyze biological triplicate samples obtained from DEG screening. In 349 and 1115 cultivars, a total of 6067 (2877 up and 3190 down) DEGs were identified at 7 days after flowering (DAF), while the up- and down-regulated DEGs at 14 DAF were 3102 and 2881. At 21 DAF, these were 1946 and 2112, respectively. Among all genes, 306 up- and 242 downregulated DEGs were expressed in both genotypes at all three stages. Furthermore, 1810 (970 up and 840 down) DEGs were expressed in both at 7 DAF and 14 DAF, and 390 (165 up and 225 down) DEGs were expressed in both strains at 7 DAF and 21 DAF. The number of stagespecifically expressed DEGs was 1436 up and 1883 down (7 DAF), 1340 up and 1428 down (14 DAF), and 989 up and 1274 down (21 DAF) (Fig. 5a-b). For genotype 349, 3003 up- and 2556 down-regulated DEGs were obtained between 7 DAF and 14 DAF, while the up- and downregulated DEGs between 7 DAF and 21 DAF were 3720 and 3642. Between 14 DAF and 21 DAF, these were 3232 and 3790, respectively. A total of 412 up- and 281 downregulated DEGs were identified common among 7 DAF, 14 DAF and 21 DAF (Fig. 5c-d). Similarly, in genotype 1115, 2613 up- and 2323 down-regulated DEGs were obtained between 7 DAF and 14 DAF, while the up- and down-regulated DEGs between 7 DAF and 21 DAF were 2323 and 1917. Between 14 DAF and 21 DAF were 3930 and 3877, respectively. A total of 685 up- and 335 downregulated DEGs were identified among 7 DAF, 14 DAF and 21 DAF (Fig. 5e-f). 


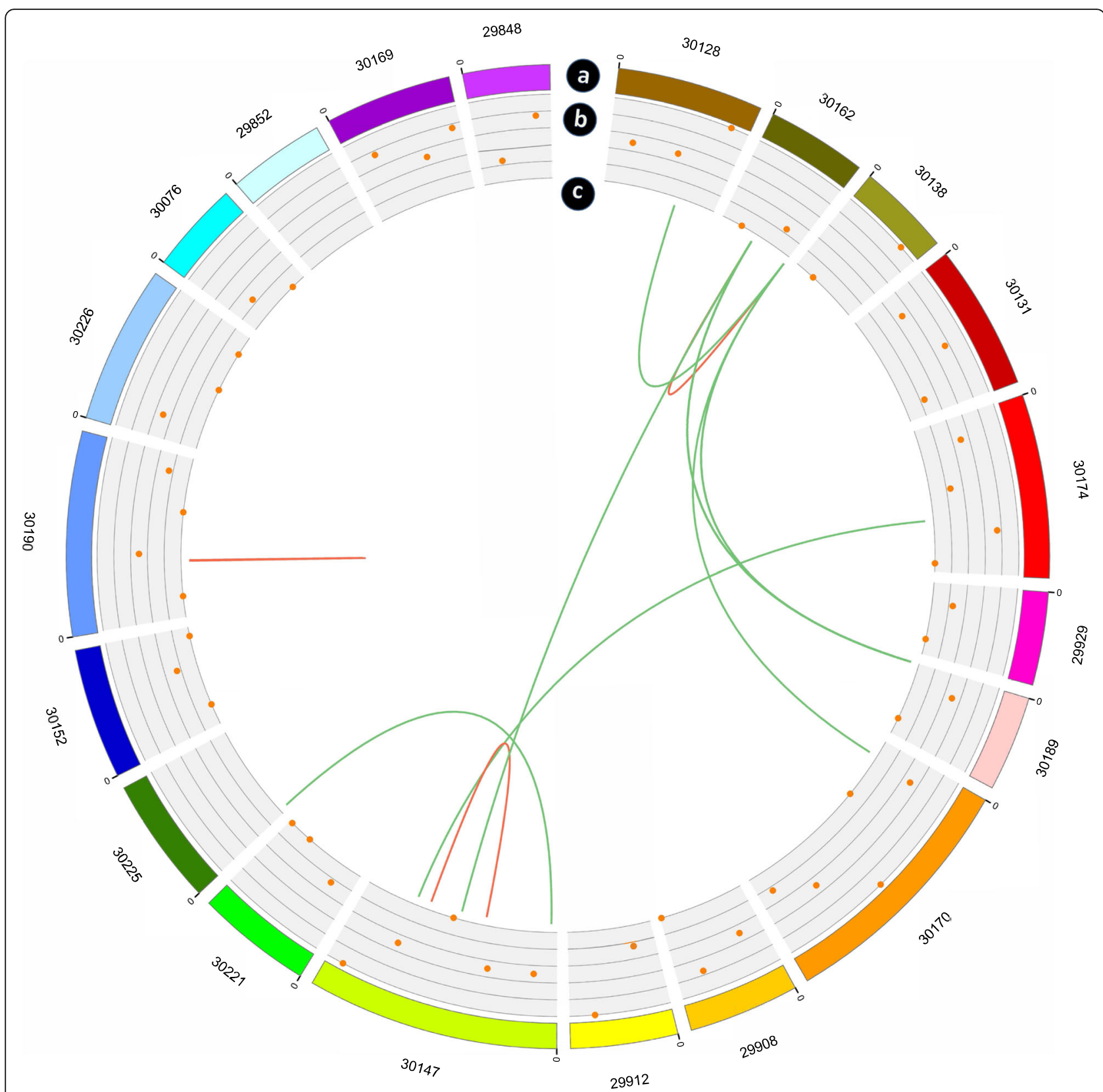

Fig. 4 CIRCOS visualization of IncRNA density and linkage of fusion transcripts for 20 top lengths of scaffolds. a Twenty longest scaffolds schematic. $\mathbf{b}$ LncRNA density, in $1 \mathrm{Mb}$ bins on each chromosome. c Linkage of fusion transcripts: red, intra-chromosomal; green, inter-chromosomal

\section{Annotation analysis of DEGs}

To better understand the function of the DEGs and further reveal the mechanism regulating ricinoleic acid biosynthesis, all DEGs were obtained in 349 and 1115 were blast with GO, COG, Pfam, KEGG, SwissProt and NR databases using Basic Local Alignment Search Tool (BLAST) software [32]. All DEGs at three time points were successfully annotated using the above mentioned seven databases (Table 1).

For the GO classification analysis, 4527, 4460 and 3037 DEGs at three time points were respectively assigned to the three main GO functional categories and then divided into 51, 50, and 47 sub-categories (Additional file 2: Figure S3). In biological process, the dominant terms at all three time points $(7,14$ and $21 \mathrm{DAF})$ were "metabolic process", "cellular process", and "single-organism process". However, "locomotion" and "biological phase" could not be detected at 21 DAF. For cellular components, "cell part", "cell", "organelle", "membrane", "membrane part", and "macromolecular complex", had accounted for the majority. The "extracellular matrix part" was not identified at 21 DAF. Regarding molecular function, the top three terms at the 


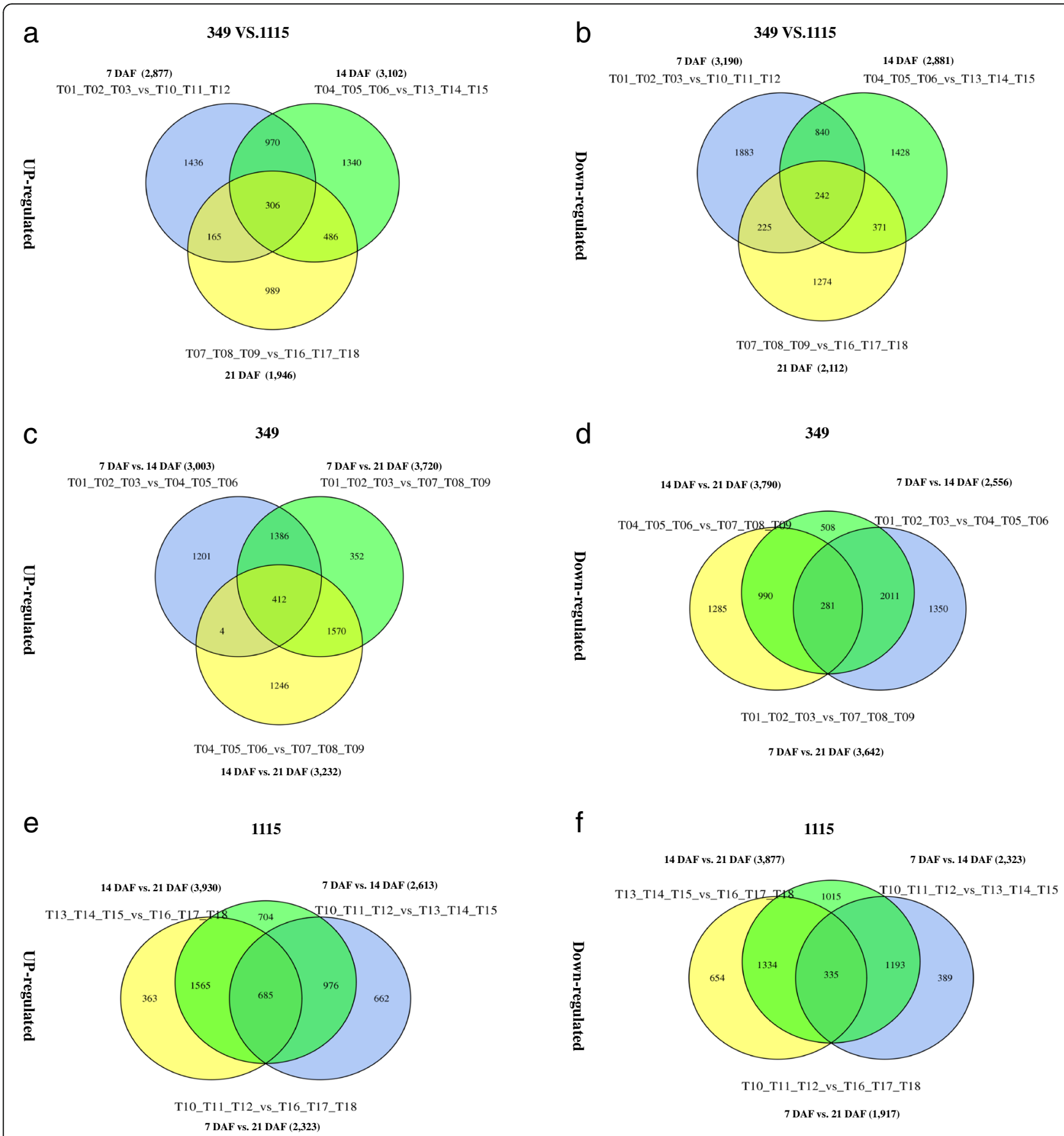

Fig. 5 Venn diagrams of differentially expressed genes (DEGs) between $R$. communis strains 349 and 1115 at three time points. a Number of up-regulated DEGs among 7 DAF, 14 DAF and 21 DAF (349 vs. 1115) (b) Number of down-regulated DEGs among 7 DAF, 14 DAF and 21 DAF. c Number of upregulated genes among 7 DAF vs. 14 DAF, 7 DAF vs. 21 DAF and 14 DAF vs. 21 DAF (349). d Number of down-regulated genes among 7 DAF vs. 14 DAF, 7 DAF vs. 21 DAF and 14 DAF vs. 21 DAF (349). e Number of up-regulated genes among 7 DAF vs. 14 DAF, 7 DAF vs. 21 DAF and 14 DAF vs. 21 DAF (1115). f Number of down-regulated genes among 7 DAF vs. 14 DAF, 7 DAF vs. 21 DAF and 14 DAF vs. 21 DAF (1115)

three time points were "binding", "catalytic activity" and "transporter activity". The term "metallochaperone activity" was only found at 7 DAF.

The transcriptome profile of metabolic pathways of immature seeds of $R$. communis is valuable for understanding the physiological processes of ricinoleic acid biosynthesis. To evaluate the metabolic differences between the two lines at three different time points, an analysis using the KEGG database on biological pathways showed that a total of 2061, 1969 and 1359 DEGs 
Table 1 Summary of annotated differentially expressed genes between R. communis strains 349 and 1115 at three time points (7 DAF, 14 DAF and 21 DAF)

\begin{tabular}{lllllllll}
\hline DEG Set & Total & COG & eggNOG & NR & Pfam & Swiss-Prot & GO & KEGG \\
\hline 349 vs. 1115 at 7 DAF & 6067 & 2391 & 5798 & 5940 & 4985 & 4449 & 4527 & 2061 \\
349 vs. 1115 at 14 DAF & 5983 & 2393 & 5719 & 5862 & 4950 & 4393 & 4460 & 1969 \\
349 vs. 1115 at 21 DAF & 4058 & 1610 & 3845 & 3963 & 3307 & 2968 & 3037 & 1359 \\
\hline
\end{tabular}

were assigned to 50 pathways (Additional file 2: Figure S4). Three pathways ("Carbon metabolism", "Biosynthesis of amino acids" and "Ribosome") were the most abundant at 7 DAF and 21 DAF, while "Carbon metabolism", "Biosynthesis of amino acids" and "Starch and sucrose metabolism" were the top three pathways at 14 DAF. Pathway enrichment analysis of DEGs is a way to indicate whether there are significant differences in a certain pathway, and the hypergeometric test [33] was used to find the pathway that was significantly enriched in the DEGs compared with the whole-genome background. The first 20 pathways with significant $p$-values are presented in Additional file 2: Figure S4. The statistics of pathway enrichment of the three time points showed that the significant differences of DEGs in pathways at 7 DAF were much higher than at 14 and 21 DAF.

In the COG database, the dominant terms at 7 DAF in "General function prediction only", were "Transcription, Replication" and "Recombination and repair". Similarly, the top three classes at 14 DAF were "Transcription, Replication", and "Signal transduction mechanisms", while three pathways such as "Signal transduction mechanisms" and "Replication, recombination and repair" were the most abundant at 21 DAF (Additional file 2: Figure S5).

\section{Identification of candidate genes related to ricinoleic acid biosynthesis and quantitative real-time reverse transcription PCR confirmation}

Ricinoleate (R), a hydroxy fatty acid (FA), has many industrial uses, including the manufacture of aviation lubricants, plastics, paints, and cosmetics. Castor oil is the only commercial source of ricinoleate. The biosynthetic pathway of triricinolein (RRR) and (12-ricinoleoylricinoleoyl) diricinoleoylglycerol (RRRR) in $R$. communis has been established [19], and the key enzymatic steps driving the conversion of ricinoleate into RRR and RRRR have been identified [19]. Several key enzymes were found to be acyl-ACP thioesterases [34], oleate 12-hydroxylase [35], diacylglycerol acyltransferase [36], phosphatidylcholine:diacylglycerol acyltransferase [37], phospholipase C2 [19], phospholipase A2 [38], lysophosphatidylcholine acyltransferase [39] and etc.. By analyzing annotated information of all DEGs between two genotypes were 349 and 1115 at the three-time points, a total of 14, 18 and 11 DEGs related to ricinoleic acid biosynthesis were identified at 7,14 and $21 \mathrm{DAF}$, respectively. Several DEGs related to the key enzymes for ricinoleic acid biosynthesis at the three-time points were selected for quantitative RT-PCR (qRT-PCR) analysis to validate the Illumina RNA sequencing data. The results showed a consistent expression trend between RNA sequencing and qRT-PCR.

At 7 DAF, 2 DEGs 29,848.t000233 (down-regulated) and 30,217.t000013 (up-regulated) had encoded acyl-ACP thioesterase enzyme. Four DEGs, 30,147.t000759 (downregulated), 30,142.t000002 (up-regulated), 29,847.t000014 (up-regulated) and 29,801.t000111 (up-regulated) related to phospholipase $\mathrm{C}$ were identified using the KEGG, NR and SwissProt databases. Only one DEG 30170.t000326 (down-regulated) encoding an enzyme with phospholipase A2 activity was annotated. Three DEGs 30,131.t000161 (down-regulated), 29,912.t000099 (down-regulated) and 29,682.t000014 (up-regulated) were found to encode diacylglycerol acyltransferases. Two DEGs 29,991.t000008 (up-regulated) and 29,706.t000035 (down-regulated) were found to encode the ratio of phosphatidylcholine:diacylglycerol acyltransferase (Table 2). The expression levels of 5 genes (29,682.t000014, 29,706.t000035, 29,756.t000030, 29,847.t000014 and 29,912.t000012) at 7 DAF were confirmed by qRT-PCR analysis to validate the Illumina RNA-seq data (Fig. 6a).

Compared 14 DAF with 7 DAF, the DEGs of key enzymes related to ricinoleic acid biosynthesis showed some interesting differences. For instance, 30,147.t000739 (down-regulated) was related to acyl-ACP thioesterase, 30,147.t000759 (up-regulated) and 29,847.t000014 (up-regulated) related to phospholipase $\mathrm{C}$ and 30,142.t000005 (down-regulated), 28, 470.t000001 (down-regulated), 29,912.t000132 (down-regulated) and 29,840.t000027 (up-regulated) were related to phospholipase A2. Moreover, 30,128.t000117 (down-regulated) encoding diacylglycerol acyltransferase was identified at 14 DAF. For DEGs related to phospholipid:diacylglycerol acyltransferase, there were no differences between 7 and 14 DAF. Only one DEG 30170.t000414 (down-regulated) encoding lysophosphatidyl acyltransferase was found at 14 DAF, while no DEG was identified at 7 DAF (Table 3). The expression levels of 6 genes $(29,840 . t 000027,29,682 . t$ 000014, 29,706.t000035, 29,847.t000014, 29,912.t000132 and 29,912.t000012) at 14 DAF were also confirmed by qRT-PCR analysis (Fig. 6b).

At 21 DAF, only one DEG, 30147.t000739 (up-regulated) related to acyl-ACP thioesterase was identified. Here, we had found a novel DEG 29756.t000030 (up- 
Table 2 List of DEGs encoding key enzymes of ricinoleic acid biosynthesis at 7 DAF

\begin{tabular}{|c|c|c|c|c|c|}
\hline Stages & Key enzyme & Gene ID & Databases & Annotation & Up/Down \\
\hline \multirow[t]{23}{*}{$7 \mathrm{DAF}$} & AAT & $29,848 . t 000233$ & KEGG & Fatty acyl-ACP thioesterase B (K10781) & Down \\
\hline & & & Pfam & Acyl-ACP thioesterase & \\
\hline & & $30,217 . t 000013$ & KEGG & Fatty acyl-ACP thioesterase B (K10782) & Up \\
\hline & & & Pfam & Acyl-ACP thioesterase & \\
\hline & & $29,756 . t 000030$ & $\mathrm{GO}$ & Phospholipase C activity (GO:0004629) & Down \\
\hline & & & KEGG & Phospholipase C (K01114) & \\
\hline & PLC & $30,147 . t 000759$ & KEGG & Phospholipase C (K01114) & Down \\
\hline & & & NR & Phospholipase C 4 precursor & \\
\hline & & $30,142 . t 000002$ & KEGG & Phospholipase C (K01114) & Up \\
\hline & & & Swiss-Prot & Phospholipase C2 (Precursor) & \\
\hline & & $29,847 . t 000014$ & NR & Phospholipase C & Up \\
\hline & & $29,801 . t 000111$ & NR & Phospholipase C & Up \\
\hline & $\mathrm{PLA}_{2}$ & $30,170 . t 000326$ & $\mathrm{GO}$ & Phospholipase A2 activity (GO:0004623) & Down \\
\hline & DGAT & $30,131 \cdot t 000161$ & Pfam & Diacylglycerol acyltransferase & Down \\
\hline & & 29,912.t000099 & KEGG & Type 1 diacylglycerol acyltransferase (K11155) & Down \\
\hline & & & NR & Type 1 diacylglycerol acyltransferase & \\
\hline & & $29,682 . t 000014$ & KEGG & Type 2 diacylglycerol acyltransferase (K14457) & Up \\
\hline & & & Pfam & Diacylglycerol acyltransferase & \\
\hline & PDAT & $29,991 . t 000008$ & KEGG & Phospholipid:diacylglycerol acyltransferase (K00679) & Up \\
\hline & & & NR & Phosphatidylcholine: Diacylglycerol Acyltransferase & \\
\hline & & $29,706 . t 000035$ & KEGG & Phospholipid:diacylglycerol acyltransferase (K00679) & Down \\
\hline & & & NR & Phosphatidylcholine: Diacylglycerol Acyltransferase & \\
\hline & & $29,912 . t 000012$ & Swiss-Prot & Phospholipid:diacylglycerol acyltransferase & Up \\
\hline
\end{tabular}

regulated) encoding phospholipase C. However, the transcript of 30,147.t000759 disappeared at 21 DAF, while 30,142.t000002 and 29,801.t000111 expressed only at 21 DAF. Compared to 14 DAF, only two DEGs, 30, 170.t000326 and 29,840.t000027 were found to encode phospholipase A2. No DEGs related to diacylglycerol acyltransferase were found at 21 DAF. Only one DEG related to phospholipid:diacylglycerol acyltransferase 29,991.t00 0008 was identified at $21 \mathrm{DAF}$. By comparison, one more DEG, 30174.t000334 (down-regulated), encoding lysophosphatidyl acyltransferase was found at 21 DAF (Table 4). The expressional levels of 4 genes $(29,840 . t 000027,29$, 847.t000014, 29,912.t000132 and 29,912.t000012) at 21 DAF were also confirmed by qPCR analysis, but not that of 29,756.t000030, whose expressional level differed from qPCR data (Fig. 6c). Moreover, a correlation analysis (Fig. 6d) of expression levels of 7 selected candidate genes related to ricinoleic acid biosynthesis at the three-time points (7, 14 and 21 DAF) showed a significant correlation (correlation coefficient $\mathrm{R}=0.749$, $p$-value $<0.05$ ) between qRT-PCR and Illumina RNA-seq, suggesting that the data generated in the RNA-seq assay of this study are of sufficiently high quality for investigating the differential expression of genes between $R$. communis two strains 349 and
1115. To better understand the complex ricinoleic acid biosynthesis processes, we constructed a putative model based on pivotal data at the transcriptional level (Fig. 7).

\section{Discussion}

The ricinoleic acid of castor oil has a great commercial value. Understanding the process of TAG assembly and the accumulation of nearly $90 \%$ of ricinoleic acid in castor oil are provided the basis of successful research on transgenic crops [19]. Similarly, the study on the basic metabolic pathways of related lipids is also conducive to the production of other highly economically valuable fatty acids through genetic engineering. It has been reported that the accumulation of ricinoleate in $R$. communis was the result of substratespecific regulation of related enzymes [19]. Several key enzymes have been identified, including AAT, FAH12, DGAT, PDAT, PLA2, PLC2 and LPCAT, which play vital roles in the fatty acid metabolism of developing castor beans. Though genetic modification of related genes, it is possible to alter the composition of fatty acids, allowing genetically modified plants to produce ricin-free seed to increases the use of plant fat as a renewable low-carbon resource. In this study, two genotypes $(R$. communis strains 349 and 1115) with contrasting castor oil content were 


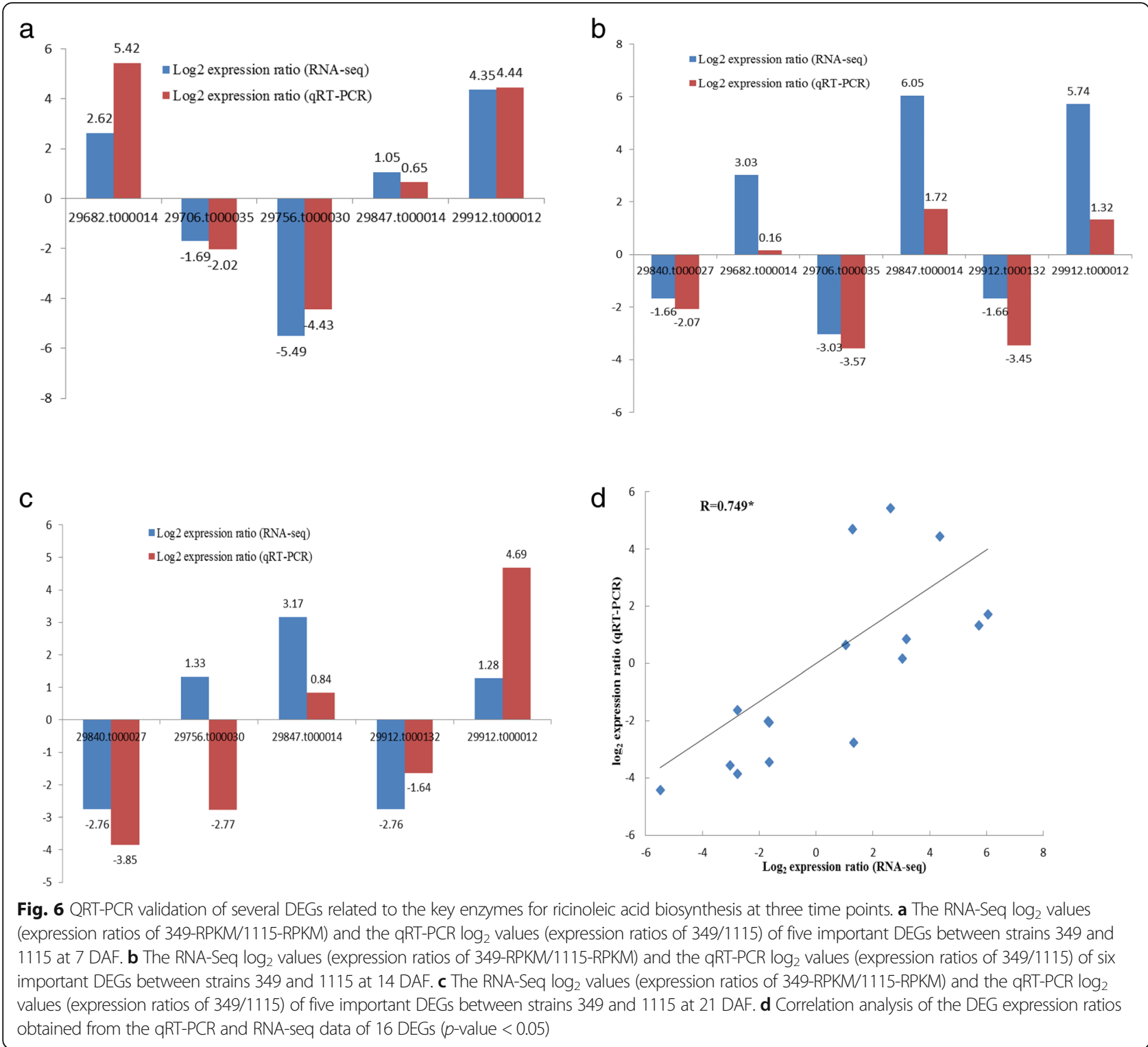

selected to better understand the ricinoleic acid biosynthesis through PacBio SMRT and Illumina RNA sequencing at three-time scales (7, 14 and 21 DAF). After transcriptome analysis, some candidate genes were identified putatively play key roles in RRR synthesis. The accumulation of lipids involves several biosynthetic and catabolic pathways, and it is impossible to significantly increase lipid content by simply expressing a specific gene [39]. Nevertheless, the cumulative expression of the relevant genes in transgenic plants may lead to higher levels of castor oil in the future.

\section{Characteristics of the genome sequence enriched with PacBio SMRT reads and analysis of LncRNAs, AS events and fusion genes}

In our study, the percentage mapped to the known nextgeneration genome had reached to $85.94 \%$. However, a total of $22.71 \%$ of isoforms detected in SMRT-seq were totally or partially mapped to the genome. Almost $62 \%$ of isoforms were potentially novel transcripts of known genes means that the information content of the enriched genome was larger than known genome. Moreover, by analyzing the 20 longest scaffolds, it was known PacBio SMRT-seq data had higher gene and transcript density than the $R$. communis reference genome. It indicates that the new transcriptome data has a great potential to improve the current version of $R$. communis annotation. A large number of new genes and exons were identified and made available for researching genes function and structure, which could provide new insights for improving castor oil content. LncRNAs are not translated into polypeptides. Nevertheless the expression and transcriptional regulation of genes is 
Table 3 List of DEGs encoding key enzymes of ricinoleic acid biosynthesis at 14 DAF

\begin{tabular}{|c|c|c|c|c|c|}
\hline Stages & Key enzyme & Gene ID & Databases & Annotation & Up/Down \\
\hline \multirow[t]{29}{*}{$14 \mathrm{DAF}$} & AAT & $29,848 . t 000233$ & KEGG & Fatty acyl-ACP thioesterase B (K10781) & Down \\
\hline & & & Pfam & Acyl-ACP thioesterase & \\
\hline & & $30,217 . t 000013$ & KEGG & Fatty acyl-ACP thioesterase B (K10782) & Up \\
\hline & & & Pfam & Acyl-ACP thioesterase & \\
\hline & & $30,147 . t 000739$ & KEGG & Fatty acyl-ACP thioesterase B (K10781) & Down \\
\hline & & & Pfam & Acyl-ACP thioesterase & \\
\hline & PLC & $30,147 . t 000759$ & KEGG & Phospholipase C (K01114) & Up \\
\hline & & & NR & Phospholipase C 4 precursor & \\
\hline & & $29,847 . t 000014$ & NR & Phospholipase C, putative & Up \\
\hline & $\mathrm{PLA}_{2}$ & $30,170.1000326$ & GO & Phospholipase A2 activity (GO:0004623) & Down \\
\hline & & $30,142 . t 000005$ & GO & Phospholipase A2 activity (GO:0004623) & Down \\
\hline & & $28,470 . t 000001$ & KEGG & Phospholipase A2 (K14674) & Down \\
\hline & & 29,912.t000132 & Pfam & Phospholipase A2 & Down \\
\hline & & & Swiss-Prot & Probable phospholipase A2 homolog 1 & \\
\hline & & $29,840 . t 000027$ & Pfam & Phospholipase A2 & Up \\
\hline & & & Swiss-Prot & Phospholipase A2-alpha (Precursor) & \\
\hline & DAGT & $30,131 \cdot t 000161$ & Pfam & Diacylglycerol acyltransferase & Down \\
\hline & & $29,912 . t 000099$ & KEGG & Type 1 diacylglycerol acyltransferase (K11155) & Down \\
\hline & & & NR & Type 1 diacylglycerol acyltransferase & \\
\hline & & $29,682 . t 000014$ & KEGG & Type 2 diacylglycerol acyltransferase (K14457) & Up \\
\hline & & & Pfam & Diacylglycerol acyltransferase & \\
\hline & & $30,128 . t 000117$ & Pfam & Diacylglycerol acyltransferase & Down \\
\hline & PDAT & $29,991 . t 000008$ & KEGG & Phospholipid:diacylglycerol acyltransferase (K00679) & Up \\
\hline & & & NR & Phosphatidylcholine: Diacylglycerol Acyltransferase & \\
\hline & & $29,706 . t 000035$ & KEGG & Phospholipid:diacylglycerol acyltransferase (K00679) & Down \\
\hline & & & NR & Phosphatidylcholine: Diacylglycerol Acyltransferase & \\
\hline & & $29,912 . t 000012$ & Swiss-Prot & Phospholipid: diacylglycerol acyltransferase & Up \\
\hline & LPCAT & $30,170 . t 000414$ & KEGG & Lysophosphatidylcholine acyltransferase (K13510) & Down \\
\hline & & & Swiss-Prot & Lysophospholipid acyltransferase & \\
\hline
\end{tabular}

significantly affected by them [40, 41]. We firstly identified lncRNAs in $R$. communis during seed development, but their functions remain unknown. Through mapping newly identified lncRNAs to the 20 longest scaffolds of $R$. communis genome, our work solidifies the conclusion that IncRNAs have a similar distribution to that of protein-coding genes and enriched outside of pericentromeric regions which has been proved in maize previously [42]. AS events are one of the key elements driving the diversity of genes and proteins in eukaryotes [43]. Although several AS events were identified in the current study highlighted the differences of castor oil contents between strains 349 and 1115 needs to be investigated further in the future. Consistent with the maize transcriptome by PacBio SMRT-seq [42], we also identified the higher proportion of inter-chromosomal (70\%) to intra-chromosomal (30\%) fusions from observing the 20 longest scaffolds. Based on these results, our work enormously had improved the existing gene models in $R$. communis and laid the foundation for future research. To insight on the structure and function of lncRNAs, AS events and fusion genes are imperative and helpful to better understand ricinoleic acid biosynthesis at the molecular level.

\section{Transcription factors}

In plants, transcription factors account for a large proportion of all proteins. There are at least 1500 transcription factors (TFs) coding genes in Arabidopsis thaliana, accounting for more than $5 \%$ of the whole genome [44]. A TF may regulate several genes related to a class of traits, thereby effectively changing the related characteristics of plants $[45,46]$. In our study, a total of 1356 TFs from 69 families were found by PacBio SMRT sequencing. For 
Table 4 List of DEGs enconding key enzymes of ricinoleic acid biosynthesis at 21 DAF

\begin{tabular}{|c|c|c|c|c|c|}
\hline Stages & Key enzyme & Gene ID & Databases & Annotation & Up/Down \\
\hline \multirow[t]{18}{*}{$21 \mathrm{DAF}$} & \multirow[t]{2}{*}{ AAT } & \multirow[t]{2}{*}{$30,147 . t 000739$} & KEGG & Fatty acyl-ACP thioesterase B (K10781) & \multirow[t]{2}{*}{ Up } \\
\hline & & & Pfam & Acyl-ACP thioesterase & \\
\hline & \multirow[t]{6}{*}{ PLC } & \multirow[t]{2}{*}{$30,142 . t 000002$} & KEGG & Phospholipase C (K01114) & \multirow[t]{2}{*}{ Up } \\
\hline & & & Swiss-Prot & Phospholipase C2 (Precursor) & \\
\hline & & $29,847 . t 000014$ & NR & Phospholipase C, putative & Up \\
\hline & & $29,801 . t 000111$ & NR & Phospholipase C, putative & Down \\
\hline & & \multirow[t]{2}{*}{$29,756 . t 000030$} & GO & Phospholipase C activity (GO:0004629) & \multirow[t]{2}{*}{ Up } \\
\hline & & & $N R$ & Phospholipase C, putative & \\
\hline & \multirow[t]{3}{*}{$\mathrm{PLA}_{2}$} & $30,170 . t 000326$ & GO & Phospholipase A2 activity (GO:0004623) & Down \\
\hline & & \multirow[t]{2}{*}{$29,840 . t 000027$} & Pfam & Phospholipase A2 & \multirow[t]{2}{*}{ Up } \\
\hline & & & Swiss-Prot & Phospholipase A2-alpha (Precursor) & \\
\hline & \multirow[t]{3}{*}{ PDAT } & \multirow[t]{2}{*}{$29,991 . t 000008$} & KEGG & Phospholipid:diacylglycerol acyltransferase (K00679) & \multirow[t]{2}{*}{ Down } \\
\hline & & & NR & Phosphatidylcholine: Diacylglycerol Acyltransferase & \\
\hline & & $29,912 . t 000012$ & Swiss-Prot & Phospholipid:diacylglycerol acyltransferase & Up \\
\hline & \multirow[t]{4}{*}{ LPCAT } & \multirow[t]{2}{*}{$30,170 . t 000414$} & KEGG & Lysophosphatidylcholine acyltransferase (K13510) & \multirow[t]{2}{*}{ Down } \\
\hline & & & Swiss-Prot & Lysophospholipid acyltransferase & \\
\hline & & \multirow[t]{2}{*}{$30,174.0000334$} & KEGG & Lysophosphatidylcholine acyltransferase (K13510) & \multirow[t]{2}{*}{ Down } \\
\hline & & & Swiss-Prot & Lysophospholipid acyltransferase & \\
\hline
\end{tabular}

instance, B3, HAP3, $b Z I P, A P 2, M Y B$ and $A R F$ regulate seed development in plants, were also found in our results. Previously, a TF called WRI1 (Wrinkled 1) has been reported to regulate TAG biosynthesis mechanism in mutant Arabidopsis seeds, which had 80\% lower TAG content than wild type [47]. In Maize, the overexpression of
WRI1 significantly increased the seed oil content by $48 \%$, and heterologous expression of the BnWRI1 gene in Arabidopsis thaliana showed increased in seed oil content by 10-40\% suggested that the WRI1 gene has a high application value for improving the oil content of plant seeds in breeding program [46]. Based on the whole-genome

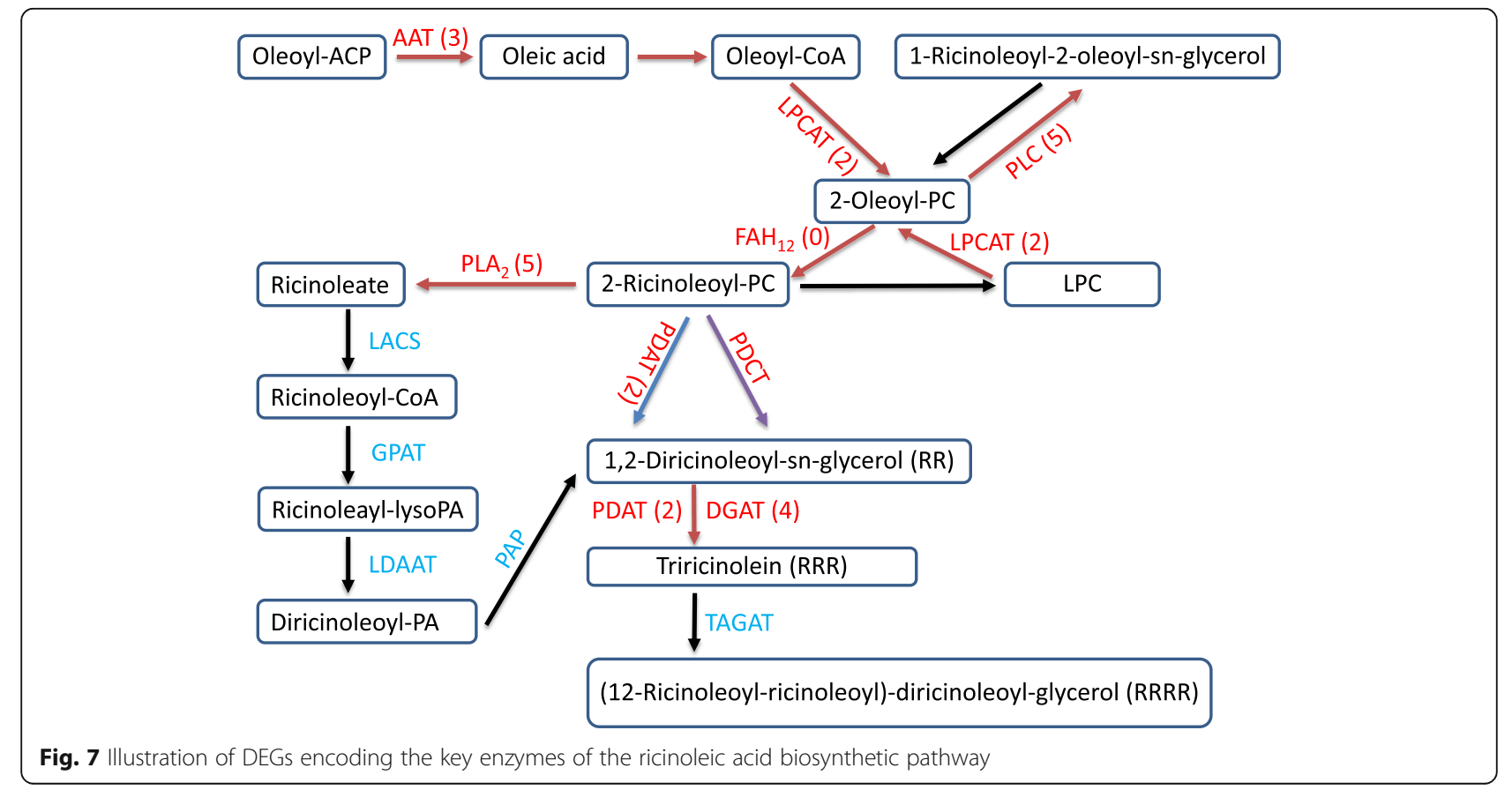


sequence of $R$. communis, Tajima et al. [47] used semiquantitative PCR and western blotting to identify several genes homologous to WRI1, which play an important role in TAG storage during seed development. Moreover, Brown et al. [3] identified several TFs such as $b H L H$, $M Y B, N A C$ etc., play important roles in ricinoleic acid metabolism, these findings were consistent to our study. Unfortunately, there were no WRI1-related genes among our results. Studying the gene expression of these abovementioned TFs will be helpful to reveal the mechanism related to the synthesis of castor oil, with important theoretical and practical significance for further improving the oil content in other crops.

\section{Conclusions}

Two genotypes with contrasting castor oil content ( $R$. communis strains 349 and 1115) were used to analyze the molecular mechanism of ricinoleic acid biosynthesis by Illumina RNA-seq and SMRT-seq. Our results significantly improved existed gene models of $R$. communis and a putative model of key genes was built to show the differences between strains 349 and 1115, illustrating the molecular mechanism of castor oil biosynthesis. A total of 22 key genes for ricinoleic acid biosynthesis were identified in the developing seeds of the two $R$. communis lines with extreme differences in oil production at three-time points. All these findings improve our knowledge of ricinoleic acid biosynthesis at the molecular level and provide a solid foundation for further studies and engineering for increasing the level of ricinoleic acid in castor beans.

\section{Methods}

\section{Plant materials and growth conditions}

The two R. communis (castor) lines 349 and 1115 with extremely different castor oil contents (43.98 and 67.51\% by Gas Chromatography/Mass Spectrometry, respectively) used in this study were obtained from National Infrastructure for Crop Germplasm Resources. Seeds were sown and cultivated in the Yangluo experimental field of the OCRI, CAAS, under standard field conditions, from spring to autumn of 2017. A total of 36 tissues (including fruit, male flower, female flower, leaf, stem and root) of the two castor lines 349 and 1115 at 7, 14 and 21 DAF were collected for PacBio SMRT sequencing. Additionally, 18 immature beans $[2$ genotypes (349 and 1115) $\times 3$ time points (7, 14 and $21 \mathrm{DAF}) \times 3$ biological replicates] were collected for Illumina RNA sequencing. Each sample from the two lines at different stages was immediately frozen and stored at $-80^{\circ} \mathrm{C}$ until further use.

\section{Total RNA extraction and library construction}

Total RNA was isolated using the TRIzol reagent (Invitrogen, Carlsbad, CA, USA) according to the manufacturer's instructions. DNase I (Promega, Madison, USA) was used to remove the residual DNA from the extracted RNA for $30 \mathrm{~min}$ at $37^{\circ} \mathrm{C}$. A NanoDrop $2000 \mathrm{UV}$-Vis spectrophotometer (NanoDrop, Wilmington, DE, USA) was used to qualify and quantify the extracted RNA, and the samples showed a 260/280 $\mathrm{nm}$ ratio between 1.8 and 2.2 and an OD260/230 > 1.0.

For obtaining the complete information of all transcripts, full length transcriptome sequencing was adopted in the present study. The best RNA sample of three replicates was selected from each of the samples and then mixed together as one sample in an equal quantity for PacBio SMRT-seq. The full-length cDNAs were synthesized using the SMRTer PCR cDNA Synthesis Kit (Biomarker, Beijing). Three SMAT cells (1-2 kb, $2-3 \mathrm{~kb}$ and $3-6 \mathrm{~kb}$ ) were run on the PacBio RSII platform (NCBI accession number: SRP131708). The resulting library was sequenced using the Iso-Seq function of PacBio RS II systems (Pacific Biosciences, Menlo Park, CA, USA) [48].

For Illumina RNA-seq, equal RNA samples at each stage of each line, including three replicates, were collected. The purity and quality of the libraries was assessed using an Agilent 2100 Bioanalyzer (Agilent, Palo Alto, California, USA) and Qubit 2.0 (Invitrogen, Carlsbad, CA, USA). Then, the 18 libraries were sequenced using the Illumina HiSeqTM 2500 sequencing platform (Illumina Inc., San Diego, CA, USA) at Biomarker Technologies Corporation in Beijing. Subsequently, the iterative isoform-clustering (ICE) algorithm was used to cluster the full-length sequences from the same isoform. Similar full-length sequences were clustered together, and each cluster had a consistent sequence.

\section{Bioinformatics and differential expression analysis}

For SMRT-seq, ROI sequences were extracted from raw reads and filtered for the cDNA primers and poly A tail. Then, the sequences were divided into full-length, nonfull-length, chimeric and non-chimeric categories according to the presence of $3^{\prime}$-primer, $5^{\prime}$-primer and poly-A (optional). Using the Quiver algorithm to cluster non-fulllength sequences, the consistent sequences were polished, and ones with high quality and low quality were obtained, respectively. The Illumina RNA-seq data were used to correct low-quality conformance sequences.

For Illumina RNA-seq, the raw RNA-seq reads were quality-checked using the FastQC (v0.11.8) to remove the adaptor sequences and low-quality reads [49]. The resulting high-quality clean reads were mapped independently to the reference genome of $R$. communis [1]. Bowtie (v2.2.3) was applied to modify the reference genome [50]. TopHat (v2.0.12) was used to align the paired-end clean reads to the reference genome [51].

Cufflinks (v2.1.1) was used to detect all transcripts using Quantitative Real-time Reverse Transcription PCR; 
[52]). Astalavista (v1.0) was applied to obtain the AS events [53]. LncTar is a tool for predicting the RNA targets of long noncoding RNAs [54]. The analysis of fusion genes and lncRNAs was performed with CPC, $\mathrm{CNCI}, \mathrm{CPAT}$ and Pfam software, according to the respective instructions. TAPIS pipeline software [55] was used to identify APA.

\section{Differential expression analysis}

The programs TopHat and Cufflinks were used to blast the sequencing reads against the reference genome of $R$. communis for the analyses of differential genes and transcript expression. It can evaluate the abundance of gene expression and also reveal new genes that have not been previously annotated using reference genomes [4]. The Fragments Per Kilobase of exon per Million fragments mapped (FPKM) method was used to calculate the abundance of gene expression. DESeq was used for analyzing biological duplicate samples obtained from DEG screening, and EBSeq [56] was used for non-biological duplicate samples. During the DEG screening, a false discovery rate (FDR) $<0.001$ and fold change $\geq 8$ were considered standard values. If the DEG fold change was $\geq 8$, then a FDR $<0.001$ was taken to indicate that the DEG was significantly different between the control and test group.

\section{Quantitative real-time reverse transcription PCR analysis}

The transcript levels of ten candidate DEGs regulating seed weight were also verified by qRT-PCR. Total RNA $(1 \mu \mathrm{g})$ was reverse transcribed using a reverse transcriptase (Takara, Japan). A $5 \mu \mathrm{l}$ aliquot of 1:20 diluted cDNA was used as the template in a $20 \mu \mathrm{l}$ PCR system. QRTPCR was performed using SYBR Green qRT-PCR Master Mix (Kapa, china), encompassing an initial denaturation step at $95^{\circ} \mathrm{C}$ for $5 \mathrm{~min}$, followed by 40 cycles of denaturation at $95^{\circ} \mathrm{C}$ for $15 \mathrm{~s}$, annealing at $60^{\circ} \mathrm{C}$ for $15 \mathrm{~s}$, and extension at $72{ }^{\circ} \mathrm{C}$ for $32 \mathrm{~s}$ in an ABI PRISM7500 Sequence Detection System (Applied Biosystems, USA). The actin gene was used as the internal standard because it is uniformly expressed in $R$. communis tissues [57]. All reactions were performed using one biological sample with three technical replicates. The comparative Ct method [58] was used for the data analysis. Primers were designed using Primer 5.0 software (Premier, Canada), and the sequences are listed in Additional file 1: Table S11.

\section{Functional annotation}

GO database was used to assign genes to broad functional categories. COG was used to classify gene products in an orthologous relationship. KOG (ftp://ftp.ncbi.nih.gov/pub/ COG/KOG/kyva) database was used to classify the homologous into different orthology clusters by combining evolutionary relationships. KEGG database was used to assign enzymes to known pathways. Pfam database was used for protein domain annotation. Overall, the functional annotation analysis of novel and differentially expressed genes was conducted by performing a blast search against the GO, COG, KEGG pathway, SwissProt and NR databases using BLAST software.

\section{Additional files}

\begin{abstract}
Additional file 1: Table S1. Statistics of sequencing data for three SMRT cells (1-2k, 2-3k and 3-6 k) by PacBio SMRT sequencing. Table S2. Statistics of reads of insert (ROI) for three SMRT cells $(1-2 k, 2-3 k$ and $3-6 k$ ) by PacBio SMRT sequencing. Table S3. Statistics of full-length reads for three SMRT cells (1-2k, 2-3k and 3-6 k) by PacBio SMRT sequencing. Table S4. Statistics of iterative clustering for error correction (ICE) for all isoforms by PacBio SMRT analysis. Table S5. The gene structure optimization information by PacBio SMRT sequencing. Table S6. The annotated information of 21,122 novel genes. Table S7. Detailed information on 250 fusion genes. Table S8. The list of 1356 TF genes. Table S9. Statistics of sequencing data for strains 349 and 1115 at three time points (7 DAF, 14 DAF and 21 DAF) by Illumina RNA sequencing. Table S10. Comparison of transcriptome sequencing reads with genome references by Illumina RNA sequencing. Table S11. The primers for quantitative real-time PCR in this study. (ZIP $1535 \mathrm{~kb}$ )

Additional file 2: Figure S1. Correlation thermograms between 18 samples from Illumina RNA sequencing. Figure S2. ROI read length distribution of each size (1-2k, 2-3k and 3-6k) of bins in the cDNA database. Figure S3. GO classification according to cellular component, molecular function and biological process of differentially expressed genes. Strain 349 vs. strain 1115 at $7 \mathrm{DAF}(\mathbf{A}), 14 \operatorname{DAF}(\mathbf{B})$, and $21 \mathrm{DAF}(\mathbf{C})$. Figure S4. KEGG classification and Pathway enrichment statistics of differentially expressed genes. (A) KEGG classification between strains 349 and 1115 at 7 DAF. (B) KEGG classification between 349 and 1115 at 14 DAF. (C) KEGG classification between 349 and 1115 at 21 DAF. (D) Pathway enrichment statistics between 349 and 1115 at 7 DAF. (E) Pathway enrichment statistics between 349 and 1115 at 14 DAF. (F) Pathway enrichment statistics between 349 and 1115 at 14 DAF. Figure S5. COG function classification of differentially expressed genes. (A) 349 vs. 1115 at 7 DAF. (B) 349 vs. 1115 at 14 DAF. (C) 349 vs. 1115 at 21 DAF. (ZIP $29130 \mathrm{~kb}$ )
\end{abstract}

\section{Abbreviations}

AAT: Oleoyl-ACP thioesterase; ACS: Acyl-CoA synthase; APA: Alternative polyadenylation; AS: Alternative splicing; BLAST: Basic local alignment search tool; CCS: Circular-consensus; CNCl: Coding-Non-Coding Index; COG: Cluster of orthologous groups of proteins; CPAT: Coding Potential Assessment Tool; CPC: Coding potential calculator; DAF: Days after flowering; DAG: Diacylglycerol; DAGT: Diacylglycerol acyltransferase; FA: Hydroxy fatty acid; FAH12: Oleoyl-12hydroxylase; FDR: False discovery rate; FL: Full-length; FPKM: Fragments Per Kilobase of exon per Million fragments mapped; GO: Gene ontology; GPAT: Glycerol-3-phosphate acyltransferase; ICE: Iterative isoform-clustering; KEGG: Kyoto Encyclopedia of Genes and Genomes; KOG: Eukaryotic ortholog groups; LACS: Long-chain lipoyl-CoA synthetase; LncRNAs: Long non-coding RNAs; LPAAT: Lysophosphatidic acid acyltransferase; LPC: Lysophosphatidylcholine; LPCAT: Lysophosphatidylcholine acyltransferase; NGS: Next-generation sequencing; NR: Non-redundant protein; PA: Phosphatidic acid; PAP: Phosphatidic acid phosphatase; PC: Phosphatidylcholine; Pfam: Protein family; PLA2: Phospholipase A2; PLC2: Phospholipase C2; QRT-PCR: Quantitative real-time reverse transcription PCR; R: Ricinoleate; $R O 1$ : Reads of insert; RRR: Triricinolein; RRRR: Diricinoleoylglycerol; SMRT: Single-molecule real-time; Swiss-Prot: The manually annotated and reviewed protein sequence database; TFs: Transcription factors; UTR: Untranslated region

\section{Acknowledgements}

We thank Biomarker Technologies Co. (Beijing, China) for providing technical support and Associate Professor Rafaqat A. Gill (Oil Crops Research Institute of the Chinese Academy of Agricultural Sciences, Wuhan, China) for English language polishing. 


\section{Authors' contributions}

$X Y$ and $X G$ designed experimental design. XG, LW and XJ performed most of experiments and wrote the manuscript. LW, WW and CF analyzed the data. All authors read and approved the final manuscript.

\section{Funding}

This work was supported by National Natural Science Foundation of China (No.31801275), Science and Technology Research Project, Science and Technology Department of Hubei Province (2018CFB310), National Program for Crop Germplasm Protection of China (2018NWB033), National Infrastructure for Crop Germplasm Resources (NICGR2018-014).

\section{Availability of data and materials}

Data for this study will be available upon publication of the manuscript; until then, the raw data can be made available to researchers upon reasonable request to the corresponding author.

\section{Ethics approval and consent to participate} Not applicable.

\section{Consent for publication}

Not applicable.

\section{Competing interests}

The authors declare that they have no competing interests.

\section{Author details}

'Oil Crops Research Institute of the Chinese Academy of Agricultural Sciences/Key Laboratory of Biology and Genetic, Improvement of Oil Crops, Ministry of Agriculture, Wuhan, China. ${ }^{2}$ Applied Biotechnology Center, Wuhan University of Bioengineering, Wuhan, China. ${ }^{3}$ College of Life Science and Technology, Henan Institute of Science and Technology/Collaborative Innovation Center of Modern Biological Breeding, Xinxiang, China.

\section{Received: 11 November 2018 Accepted: 23 May 2019}

Published online: 06 June 2019

\section{References}

1. Chan AP, Crabtree J, Zhao Q, Lorenzi H, Orvis J, Puiu D, et al. Draft genome sequence of the oilseed species Ricinus communis. Nat Biotechnol. 2010;28:951-6.

2. Gill RA, Ali B, Cui P, Shen E, Farooq MA, Islam F, et al. Comparative transcriptome profiling of two Brassica napus cultivars under chromium toxicity and its alleviation by reduced glutathione. BMC Genomics. 2016;17:885.

3. Brown AP, Kroon JT, Swarbreck D, Febrer M, Larson TR, Graham IA, et al. Tissuespecific whole transcriptome sequencing in castor, directed at understanding triacylglycerol lipid biosynthetic pathways. PLoS One. 2012;7:1-13.

4. Geng X, Dong N, Wang Y, Li G, Wang L, Guo X, et al. RNA-seq transcriptome analysis of the immature seeds of two Brassica napus lines with extremely different thousand-seed weight to identify the candidate genes related to seed weight. PLoS One. 2018;13:e0191297.

5. Li Y, Fang C, Fu Y, Hu A, Li C, Zou C, et al. A survey of transcriptome complexity in Sus scrofa using single-molecule long-read sequencing. DNA Res. 2018;25:421-37.

6. Secco D, Jabnoune M, Walker $H$, Shou H, Wu P, Poirier $Y$, et al. Spatiotemporal transcript profiling of rice roots and shoots in response to phosphate starvation and recovery. Plant Cell. 2013;25:4285-304.

7. Lan P, Li W, Schmidt W. Complementary proteome and transcriptome profiling in phosphate-deficient Arabidopsis roots reveals multiple levels of gene regulation. Mol Cell Proteomics. 2012;11:1156-66.

8. Oono $Y$, Kawahara $Y$, Yazawa $T$, Kanamori H, Kuramata M, Yamagata $H$, et al. Diversity in the complexity of phosphate starvation transcriptomes among rice cultivars based on RNA-Seq profiles. Plant Mol Biol. 2013;83:523-37.

9. Li Z, Xu C, Li K, Yan S, Qu X, Zhang J. Phosphate starvation of maize inhibits lateral root formation and alters gene expression in the lateral root primordium zone. BMC Plant Biol. 2012;12:89.

10. Du H, Yu Y, Ma Y, Gao Q, Cao Y, Chen Z, et al. Sequencing and de novo assembly of a near complete indica rice genome. Nat Commun. 2017;8:15324.

11. Madeleine C, Andrey AY, Julian JA, Colin EA, Pawel H, Kathryn RE. Correction to: De novo transcriptome assembly, annotation and comparison of four ecological and evolutionary model salmonid fish species. BMC Genomics. 2018;19:448.
12. Metzker ML. Sequencing technologies-the next generation. Nat rev Genet 2010;11:31-46.

13. Tilgner H, Jahanbani F, Blauwkamp T, Moshrefi A, Jaeger E, Chen F, et al. Comprehensive transcriptome analysis using synthetic long-read sequencing reveals molecular co-association of distant splicing events. Nat Biotechnol. 2015;33:736-42.

14. Eid J, Fehr A, Gray J, Luong K, Lyle J, Otto G, et al. Real-time DNA sequencing from single polymerase molecules. Science. 2009;323:133-8.

15. Koren S, Schatz MC, Walenz BP, Martin J, Howard J, Ganapathy G, et al. Hybrid error correction and de novo assembly of single-molecule sequencing reads. Nat Biotechnol. 2012;30:693-700.

16. Sharon D, Tilgner $H$, Grubert F, Snyder M. A single-molecule long-read survey of the human transcriptome. Nat Biotechnol. 2013;31:1009-14.

17. Ren P, Meng Y, Li B, Ma X, Si E, Lai Y, et al. Molecular mechanisms of acclimatization to phosphorus starvation and recovery underlying full-length transcriptome profiling in barley (Hordeum vulgare L.). Front Plant Sci. 2018;9:500.

18. Xu Z, Peters RJ, Weirather J, Luo H, Liao B, Zhang X, et al. Full-length transcriptome sequences and splice variants obtained by a combination of sequencing platforms applied to different root tissues of Salvia miltiorrhiza and tanshinone biosynthesis. Plant J. 2015;82:951-61.

19. Lin J, Arcinas A. Regiospe analysis of diricinoleoyla cylg-lycerols in castor (Ricinus communis L.) oil by electrospray lonization-mass spectrometry. J Agric Food Chem. 2007:55:2209-16.

20. Sujatha M. Biotechnological interventions for improving jatropha and castor for biofuels. Petrotech. 2009;11:864-9.

21. Tatusov RL, Galperin MY, Natale DA, Koonin EV. The COG database: a tool for genome-sale analysis of protein functions and evolution. Nucleic Acids Res. 2000;28:33-6.

22. Ashburner M, Ball CA, Blake JA, Botstein D, Butler H, Cherry JM, et al. Gene ontology: tool for the unification of biology. The gene ontology consortium. Nat Genet. 2000;25:25-9.

23. Kanehisa M, Goto S, Kawashima S, Okuno Y, Hattori M. The KEGG resource for deciphering the genome. Nucleic Acids Res. 2004;32:277-80.

24. Punta M, Coggill PC, Eberhardt RY, Mistry J, Tate J, Boursnell C, et al. The Pfam protein families database. Nucleic Acids Res. 2011;40:290-301.

25. Apweiler R, Bairoch A, Wu CH, Barker WC, Boeckmann B, Ferro S, et al. UniProt: the universal protein knowledgebase. Nucleic Acids Res. 2004;32:115-9.

26. Deng YY, Li JQ, Wu SF, Zhu YP, Chen YW, He FC. Integrated nr database in protein annotation system and its localization. Comput Eng. 2006;32:71-4.

27. Weirather JL, Afshar PT, Clark TA, Tseng E, Powers LS, Underwood JG, et al. Characterization of fusion genes and the significantly expressed fusion isoforms in breast cancer by hybrid sequencing. Nucleic Acids Res. 2015;43:116.

28. Kong L, Zhang Y, Ye ZQ, Liu XQ, Zhao SQ, Wei L, et al. CPC: assess the proteincoding potential of transcripts using sequence features and support vector machine. Nucleic Acids Res. 2007;35:345-9.

29. Sun L, Luo H, Bu D, Zhao G, Yu K, Zhang C, et al. Utilizing sequence intrinsic composition to classify protein-coding and long non-coding transcripts. Nucleic Acids Res. 2013;41:166.

30. Wang L, Park HJ, Dasari S, Wang S, Kocher J, Li W. CPAT: coding-potential assessment tool using an alignment-free logistic regression model. Nucleic Acids Res. 2013;41:74.

31. Wang L, Feng Z, Wang X, Wang X, Zhang X. DEGseq: an R package for identifying differentially expressed genes from RNA-seq data. Bioinformatics. 2010;26:136-48.

32. Altschul SF, Madden TL, Schäffer AA, Zhang J, Zhang Z, Miller W, et al. Gapped BLAST and PSIBLAST: a new generation of protein database search programs. Nucleic Acids Res. 1997:25:3389-402.

33. Stathias V, Pastori C, Griffin TZ, Komotar R, Clarke J, Zhang M, et al. Identifying glioblastoma gene networks based on hypergeometric test analysis. PLoS One 2014;9:e115842.

34. Sanchez-Garcia A, Moreno-Perez AJ, Muro-Pastor AM, Salas JJ, Garces R, Martinez-Force E. Acyl-ACP thioesterases from castor (Ricinus communis L.): an enzymatic system appropriate for high rates of oil synthesis and accumulation. Phytochemistry. 2010;71:860-9.

35. Van de Loo FJ, Broun P, Turner S, Somerville C. An oleate 12-hydroxylase from Ricinus communis L. is a fatty acyl desaturase homolog. Proc Natl Acad Sci U S A. 1995;92:6743-7.

36. Burgal J, Shockey J, Lu C, Dyer J, Larson T, Graham L, et al. Metabolic engineering of hydroxy fatty acid production in plants: RCDGAT2 drives dramatic increases in ricinoleate levels in seed oil. Plant Biotechnol J. 2008;6:819-31. 
37. Kim HU, Lee KR, Go YS, Jung JH, Suh M, Kim JB. Endoplasmic reticulumlocated PDAT1-2 from castor bean enhances hydroxy fatty acid accumulation in transgenic plants. Plant Cell Physiol. 2011;52:983-93.

38. Lin JT, Woodruff CL, Lagouche OJ, Mckeon TA, Stafford AE. Biosynthesis of triacylglycerols containing ricinoleate in castor micrsomes using 1-acyl-2oleoyl-sn-glycerol-3-phos-phocholine as the substrate of oleoyl-12hydroxylase. Lipids. 1998;33:59-69.

39. Arroyo-Caro JM, Chileh T, Alonso DL, Garcia-Maroto F. Molecular characterization of a lysophosphatidylcholine acyltransferase gene belonging to the MBOAT family in Ricinus communis L. Lipids. 2013;48:663-74.

40. Batista PJ, Chang HY. Long noncoding RNAs: cellular address codes in development and disease. Cell. 2013;152:1298-307.

41. Iyer MK, Niknafs YS, Malik R, Singhal U, Sahu A, Hosono Y, et al. The landscape of long noncoding RNAs in the human transcriptome. Nat Genet. 2015;47:199-208

42. Wang B, Tseng E, Regulski M, Clark TA, Hon T, Jiao Y, et al. Unveiling the complexity of the maize transcriptome by single-molecule long-read sequencing. Nat Commun. 2016;7:11708.

43. Gao Y, Wang J, Zheng Y, Zhang J, Chen S, Zhao F. Comprehensive identification of internal structure and alternative splicing events in circular RNAs. Nat Commun. 2016;7:12060.

44. Riechmann JL, Heard J, Martin G, Reuber L, Jiang CZ, Keddie J, et al. Arabidopsis transcription factors: genome-wide comparative analysis among eukaryotes. Science. 2000;290:2105-10.

45. Tian XR, Li SD, Liu YS, Liu XM. Transcriptomic profiling reveals metabolic and regulatory pathways in the desiccation tolerance of mungbean (Vigna radiata [L.] R. Wilczek). Front Plant Sci. 2016;7:1921.

46. Wang A, Hu J, Huang X, Li X, Zhou G, Yan Z. Comparative transcriptome analysis reveals heat-responsive genes in Chinese cabbage Brassica rapa ssp. chinensis. Front Plant Sci. 2016;7:939.

47. Tajima D, Kaneko A, Sakamoto M, Ito Y, Hue NT, Miyazaki M, et al. Wrinkled (WR/1) homologs, AP2-type transcription factors involving master regulation of seed storage oil synthesis in castor bean (Ricinus communis L.). American J Plant Sci. 2013:4:333-9.

48. Lan T, Renner T, Ibarra-Laclette E, Farr KM, Chang TH, Cervantes-Pérez SA, et al. Long-read sequencing uncovers the adaptive topography of a carnivorous plant genome. Proc Natl Acad Sci. 2017:14:4435-41.

49. Zhu C, Li X, Zheng J. Transcriptome profiling using Illumina- and SMRTbased RNA-seq of hot pepper for in-depth understanding of genes involved in CMV infection. Gene. 2018;666:123-33.

50. Langmead B, Salzberg SL. Fast gapped-read alignment with bowtie 2. Nat Methods. 2012:9:357.

51. Trapnell C, Pachter L, Salzberg SL. TopHat: discovering splice junctions with RNA-Seq. Bioinformatics. 2009;25:1105-11.

52. Trapnell C, Roberts A, Goff L, Pertea G, Kim D, Kelley DR, et al. Differential gene and transcript expression analysis of RNA-seq experiments with TopHat and cufflinks. Nat Protoc. 2012;7:562.

53. Foissac S, Sammeth M. ASTALAVITA: dynamic and flexible analysis of alternative splicing events in custom gene datasets. Nucleic Acids Res. 2007;35:297-9.

54. Li J, Ma W, Zeng P, Wang J, Geng B, Yang J, et al. LncTar: a tool for predicting the RNA targets of long noncoding RNAs. Brief Bioinform. 2015;16:806.

55. Abdelghany SE, Hamilton M, Jacobi JL, Ngam P, Devitt N, chilkey F. A survey of the sorghum transcriptome using single-molecule long reads. Nat Commun. 2016;7:11706.

56. Leng N, Dawson JA, Thomson JA, Ruotti V, Rissman Al, Smits BM, et al. EBSeq: an empirical Bayes hierarchical model for inference in RNA-seq experiments. Bioinformatics. 2013;29:1035-43.

57. Wang X, Wang L, Yan X, Wang L, Tan M, Geng X, et al. Transcriptome analysis of the germinated seeds identifies low-temperature responsive genes involved in germination process in Ricinus communis. Acta Physiol Plant. 2016;38:6

58. Schmittgen TD, Livak KJ. Analyzing real-time PCR data by the comparative $C_{\text {T }}$ method. Nat Protoc. 2008:3:1101-8.

\section{Publisher's Note}

Springer Nature remains neutral with regard to jurisdictional claims in published maps and institutional affiliations.

Ready to submit your research? Choose BMC and benefit from:

- fast, convenient online submission

- thorough peer review by experienced researchers in your field

- rapid publication on acceptance

- support for research data, including large and complex data types

- gold Open Access which fosters wider collaboration and increased citations

- maximum visibility for your research: over $100 \mathrm{M}$ website views per year

At BMC, research is always in progress.

Learn more biomedcentral.com/submissions 